\title{
Outcome of pregnancy in patients with autoimmune rheumatic disease before the disease onset
}

\author{
A SIAMOPOULOU-MAVRIDOU, M N MANOUSSAKIS, A K MAVRIDIS, \\ AND H M MOUTSOPOULOS
}

From the Department of Medicine, Medical School, University of Ioannina, 45332 Ioannina, Greece $\vec{\circ}$

SUMMARY The outcomes of 419 pregnancies of 154 unselected patients with various autoe immune diseases, including 390 pregnancies before the disease onset, were studied retrospect tively. The patients comprised 40 with systemic lupus erythematosus (SLE), 72 with rheumatoid arthritis, 21 with primary Sjögren's syndrome (1 $\left.{ }^{\circ} \mathrm{SS}\right), 14$ with progressive systemic sclerosiso (PSS), and seven with mixed connective tissue disease. The histories of 267 pregnancies of 980 healthy, age matched women served as controls. Our data indicate that compared with health $\$$ controls autoimmune patients do not experience a higher incidence of fetal loss. The incidence of fetal loss before disease onset in the various groups of autoimmune patients (as well as afted disease onset in patients with SLE and RA) was not significantly different from that of controlse Spontaneous abortions in patients with $1^{\circ} \mathrm{SS}$ and PSS before disease onset occurred significantl部 more frequently $(p<0 \cdot 05)$ than in controls. Nevertheless, it should be noted that this was not the case when the incidence per woman was considered. On the other hand, patients with SLE, bett before and after disease onset, experienced a higher incidence of premature deliveries $(\mathrm{p}<0.05)^{00}$ Finally, the analysis of autoantibody profiles, including antibodies to nuclear antigens, ב्गे Ro(SSA) cellular antigen, to double stranded DNA, and to cardiolipin, could not demonstrate any association of autoantibodies with any particular pregnancy outcome.

Key words: fetal loss, autoantibodies, anticardiolipin antibodies.

Autoimmune rheumatic diseases (ARD) are known to occur predominantly in women. Fertility in patients with ARD does not seem to be impaired, ${ }^{1}$ therefore pregnancy in these individuals is frequently of clinical relevance.

Several studies have shown that spontaneous abortion and premature deliveries are frequently encountered in the pregnancies of women with systemic lupus erythematosus (SLE). ${ }^{1-3}$ Pregnancy in SLE has been extensively studied owing to the fact that this disease affects primarily women of childbearing age. In contrast, data on pregnancy outcome in certain other autoimmune diseases, such as primary Sjögren's syndrome $\left(1^{\circ} \mathrm{SS}\right)$ or progressive systemic sclerosis (PSS), are as yet inadequate, presumably because these entities mainly occur in women during the fourth or the fifth decade of

Accepted for publication 20 May 1988.

Correspondence to $\mathrm{Dr}$ A Siamopoulou-Mavridou, Department of Medicine, Medical School, University of Ioannina, 45332 Ioannina, Greece. life. $^{45}$ Furthermore, there is some controvers between previously published studies regarding whether patients with SLE already present a highe incidence of complicated pregnancies before thef disease onset. ${ }^{36}$

The increased incidence of fetal loss in patients with SLE has been attributed to the presence of placental vasculitis and infarctions. Further, mate nal autoantibody responses, including immune con plex deposition and the cross reaction of lymph cytotoxic antibodies with trophoblast, have been incriminated. ${ }^{7}$ The recently presented associatiof between the presence of anticardiolipin (anti-CL) antibodies and habitual abortions in patients wi SLE is currently receiving considerable attention. ${ }^{808}$ Antibodies reacting with cardiolipin and other anionic phospholipids have also been associat with the presence of vascular thrombosis in patien with SLE and SLE-like disease. ${ }^{8}$ Such anti-CI associated thrombotic events are believed to cause placental infarctions and subsequent fetal loss these patients. ${ }^{8}$ 
The main purpose of this study was to investigate whether patients with autoimmune diseases suffer from an increased incidence of complicated pregnancies before the development of clinical manifestations. For this purpose, the histories of pregnancies both before and after the disease onset of a fairly large number of unselected patients with various autoimmune diseases were retrospectively studied. Further, we evaluated the autoantibody profiles of these patients, including anti-CL antibodies, with respect to the outcome of their pregnancies.

\section{Patients and methods}

The histories of 419 pregnancies in 154 consecutive unselected Greek married women with various autoimmune rheumatic diseases were retrospectively evaluated. Patients in this study included 72 women with classical rheumatoid arthritis (RA), ${ }^{10}$ 40 with SLE,${ }^{10} 21$ with $1^{\circ} S S,{ }^{4} 14$ with PSS, ${ }^{5}$ and seven with mixed connective tissue disease (MCTD) ${ }^{3}$ Patients were specifically interviewed for the number of pregnancies, full terms, premature deliveries (spontaneous termination of gestation between the 21st and 37th week), stillbirths (intrauterine fetal death after 21st week of gestation and before birth), and spontaneous abortions (interruption of pregnancy before 20 weeks of gestation). In addition, fetal loss (sum of spontaneous abortions and stillbirths) and fertility rate (average number of pregnancies per individual) were estimated. ${ }^{3}$ Control material consisted of the histories of 267 pregnancies of 98 healthy Greek women visiting the outpatient gynaecological clinic routinely, matched for age and for socioeconomic and marital status with the patients studied. In addition, serum samples from these healthy individuals were used as controls in serological studies. We also retrospectively evaluated the autoantibody profile of patients during their first assessment in our clinic, at a time ranging from 0 to 12 years after their disease onset (mean (SD) for SLE 2.5 (2.0) years, RA 3.1 (3.0) years, $1^{\circ}$ SS $3.8(3.5)$ years, PSS $3.2(2.9)$ years, and MCTD $1.3(0.8)$ years after disease onset). Autoantibody profiles consisted of antibodies to nuclear antigens (ANAs, detected by indirect immunofluorescence using $\mathrm{HEp}_{2}$ epithelial cells as substrate, positive ANA titre 1/80), antibodies to Ro(SSA) cellular antigen (by counterimmunoelectrophoresis and double immunodiffusion using human spleen and calf thymus extracts), as well as of IgG antibodies to double stranded DNA) (anti-dsDNA) and IgG and IgM antibodies to cardiolipin (anti-CL) by isotype specific, enzyme linked immunosorbent assays (ELISAs), as previously described. ${ }^{11} 12$
Results in anti-dsDNA and anti-CL assays were expressed as a binding index (BI), as previously. ${ }^{13} \mathrm{~A}$ binding index of $100(\mathrm{BI}=100)$ was defined as the cut off point for positivity (four standard deviations above the mean of a large number of healthy blood donors previously examined). The presence of the above autoantibodies was also examined in the serum samples from the 98 healthy women (controls).

STATISTICAL ANALYSIS

Data were analysed by $\chi^{2}$ test with Yates's correction and Student's $t$ test, where applicable.

\section{Results}

No differences were observed between the fertility rates of the healthy control group $(2.7)$ and the various groups of patients studied (ranging from 2.5 to $3 \cdot 0)$.

In the various groups of patients 390 out of the 419 pregnancies had ended before the disease onset (Tables 1,2). The time between the above pregnancies and the disease onset ranged between 1 and 45 years, and no differences were observed in this between the various groups of patients. Twenty nine additional pregnancies (Table 1) occurred-14 in 12 patients with SLE and 15 in 10 patients with RA - after the onset of the disease (two months up to four years). Tables 1 and 2 show the incidences of the various outcomes of pregnancies in patients and controls.

Recurrent (more than one, ranging from two to four) spontaneous abortions occurred in 18 patients with ARD (two with SLE, nine with RA, three each with $1^{\circ} S S$ and PSS, and one with MCTD) and three healthy women, while recurrent stillbirths occurred in four patients with ARD (two with SLE and one each with RA and $1^{\circ} \mathrm{SS}$ ).

A comparison of the pregnancy outcome in the various patients groups before the development of the disease and in the controls did not show any remarkable differences (Tables 1 and 2). The incidence of fetal loss was found to be similar in all groups examined. Patients with $1^{\circ} \mathrm{SS}$ and PSS had a higher incidence of spontaneous abortions (per pregnancy) than controls $(p<0.05)$ (Table 2$)$. On the other hand, prematurity was significantly more frequent in patients with SLE than in controls (per pregnancy, as well as per woman, $p<0.05$ ). Stillbirths in pregnancies of patients with SLE were significantly more frequent after than before disease onset $(p<0.05)($ Table 1). Further, although the difference was not statistically significant, the incidence of prematurity increased in patients with SLE after disease onset. 
Table 1 Outcome of pregnancy before and after the clinical onset of systemic lupus erythematosus (SLE) and rheumatoid arthritis $(R A)$

\begin{tabular}{|c|c|c|c|c|c|}
\hline & \multicolumn{2}{|c|}{$S L E \quad(n=40)$} & \multicolumn{2}{|c|}{$R A(n=72)$} & \multirow{2}{*}{$\begin{array}{l}\text { Controls } \\
(n=98) \\
\frac{\bar{C}}{\bar{C}}\end{array}$} \\
\hline & $\begin{array}{l}\text { Before } \\
(n=38)\end{array}$ & $\begin{array}{l}\text { After } \\
(n=12)\end{array}$ & $\begin{array}{l}\text { Before } \\
(n=69)\end{array}$ & $\begin{array}{l}\text { After } \\
(n=10)\end{array}$ & \\
\hline $\begin{array}{l}\text { Mean age } \dagger(\mathrm{SD}) \\
\text { (range) } \\
\text { No of pregnancies } \\
\text { No of full terms } \ddagger(\%) \\
\text { No of premature births (\%) } \\
\text { No of spontaneous abortions (\%) } \\
\text { No of stillbirths (\%) } \\
\text { No of fetal losses (\%) }\end{array}$ & $\begin{array}{l}43(13) \\
(25-75) \\
81 \\
69(85) \\
4(5)^{*} \\
7(9) \\
1(1) \\
8(10)\end{array}$ & $\begin{array}{l}26(8) \\
(19-40) \\
14 \\
11(79) \\
1(7)^{*} \\
0(0) \\
2(14)^{* *} \\
2(14)\end{array}$ & $\begin{array}{l}43(12) \\
(19-69) \\
191 \\
155(81) \\
2(1) \\
30(16) \\
4(2) \\
34(18)\end{array}$ & $\begin{array}{l}23(5) \\
(19-38) \\
15 \\
11(73) \\
0(0) \\
4(27) \\
0(0) \\
4(27)\end{array}$ & 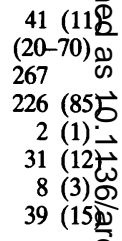 \\
\hline \multicolumn{6}{|c|}{$\begin{array}{l}{ }^{*} \mathrm{p}<0.05 \text { compared with control; }{ }^{* *} \mathrm{p}<0.05 \text { compared with data from patients with SLE before disease onset. } \\
\text { †Mean age (years) at disease onset. } \\
\text { †Newborns, small for gestational age, were one found in SLE and the RA groups. }\end{array}$} \\
\hline \multicolumn{6}{|c|}{$\begin{array}{l}\left.\text { Table } 2 \text { Outcome of pregnancy before the clinical onset of primary Sjögren's syndrome (1 }{ }^{\circ} S S\right) \text {, progressive systemic } \\
\text { sclerosis (PSS), and mixed connective tissue disease (MCTD) }\end{array}$} \\
\hline & $\begin{array}{l}1^{\circ} S S \\
(n=21)\end{array}$ & $\begin{array}{l}P S S \\
(n=14)\end{array}$ & & $\begin{array}{l}M C T D \\
(n=7)\end{array}$ & $\begin{array}{l}\text { Controls } \\
(n=98)\end{array}$ \\
\hline $\begin{array}{l}\text { Mean age } †(\mathrm{SD}) \\
\text { (range) }\end{array}$ & $\begin{array}{l}48(9) \\
(35-69)\end{array}$ & $\begin{array}{l}45(11) \\
(30-73)\end{array}$ & & $\begin{array}{l}38(12) \\
(23-60)\end{array}$ & $\begin{array}{c}41(\mathrm{q}) \\
(20-1)\end{array}$ \\
\hline No of pregnancies & 63 & 36 & & 19 & 267 은. \\
\hline No of full terms (\%) & $48(76)$ & $28(78)$ & & $16(84)$ & $226(8 \overline{57})$ \\
\hline No of premature births (\%) & $0(0)$ & $0(0)$ & & $0(0)$ & $2(1)$ \\
\hline No of spontaneous abortions (\%) & $13(21)^{*}$ & $8(22)^{*}$ & & $3(16)$ & $31(12)$ \\
\hline No of stillbirths (\%) & $2(3)$ & $0(0)$ & & $0(0)$ & $8(3)$ \\
\hline No of fetal losses (\%) & $15(24)$ & $8(22)$ & & $3(16)$ & $39(15)$ 용 \\
\hline
\end{tabular}

${ }^{*} \mathrm{p}<0.05$ compared with control.

†Mean age (years) at disease onset.

When data for patients with RA before and after disease onset were compared, an increase (not statistically significant) in the incidence of spontaneous abortions was noted (Table 1).

Most patients studied were serologically hyperreactive, as exemplified by the incidence of positive ANA tests (in $70 \%$ of patients, mean positive ANA titre 1/905), anti-Ro(SSA) antibodies (in $25 \%$ of patients), IgG anti-CL (in $10 \%$, mean positive $\mathrm{BI}=235$ ), and $\mathrm{IgM}$ anti-CL (in $5 \%$, mean positive $B I=343)$. Finally, more than half $(55 \%)$ of the patients with SLE had anti-dsDNA antibodies (mean positive $\mathrm{BI}=413$ ) (Table 3 ). In the healthy control population positive ANA tests were evident in three women (titre 1/320), while IgG anti-CL were found in another three (with $\mathrm{BI}=143,197$, and 111 respectively) and IgM anti-CL in one $(B I=132)$ (Table 3). We could not show any association between the outcome of pregnancy and the presence of any particular autoantibody under considerationo This was still the case when data from women olde? than 40 years at the time of serum collection were omitted, in an attempt to exclude the possibility of age induced autoantibodies ${ }^{13}$ (data not shown). The occurrence of autoantibodies before the disease onset could not be verified, however. Therefore, we further examined the correlation of autoantibod胥. profiles with the outcome of pregnancies in the 120 patients with SLE and the 10 patients with RA with pregnancies after the disease onset (14 and 1今 pregnancies respectively), where serum samples before gestation were available. Antibodies coul $\phi$ still not be associated with any of the various pregnancy outcomes (Table 4). Six pregnancie ended with fetal loss; anti-Ro(SSA) and ant? dsDNA antibodies were present in two of themo while anti-CL antibodies were detected in one. IT addition, a premature delivery was experienced by $\mathbb{\infty}$ 
Table 3 Autoantibody profile of patients and controls

\begin{tabular}{lllllll}
\hline $\begin{array}{l}\text { Serological } \\
\text { parameters }\end{array}$ & $\begin{array}{l}S L E \\
(n=40)\end{array}$ & $\begin{array}{l}R A \\
(n=72)\end{array}$ & $\begin{array}{l}1^{\circ} S S \\
(n=21)\end{array}$ & $\begin{array}{l}P S S \\
(n=14)\end{array}$ & $\begin{array}{l}M C T D \\
(n=7)\end{array}$ & $\begin{array}{l}\text { Controls } \\
(n=98)\end{array}$ \\
\hline ANA & $35(88)^{*}$ & $35(49)$ & $17(81)$ & $14(100)$ & $6(86)$ & $3(3)$ \\
Anti-Ro(SSA) & $23(58)$ & $7(10)$ & $7(33)$ & $1(7)$ & $1(14)$ & $0(0)$ \\
IgG anti-dsDNA & $22(55)$ & $0(0)$ & $0(0)$ & $0(0)$ & $0(0)$ & $0(0)$ \\
IgG anti-CL & $10(25)$ & $4(6)$ & $1(5)$ & $0(0)$ & $0(0)$ & $3(3)$ \\
IgM anti-CL & $4(10)$ & $1(1)$ & $2(10)$ & $0(0)$ & $1(14)$ & $1(1)$ \\
\hline
\end{tabular}

${ }^{*}$ Values are No (\%).

SLE=systemic lupus erythematosus; RA=rheumatoid arthritis; $1^{\circ} \mathrm{SS}=$ primary Sjögren's syndrome; PSS=progressive systemic sclerosis; $\mathrm{MCTD}=$ mixed connective tissue disease; $\mathrm{ANA}=$ antinuclear antibody; anti-CL=anticardiolipin.

Table 4 Correlation of the presence of antibodies to $R o(S S A), d s D N A$, and cardiolipin (CL) with the incidence of complicated pregnancies in patients after the disease onset

\begin{tabular}{|c|c|c|c|c|c|c|}
\hline & \multicolumn{2}{|c|}{ SLE (14 pregnancies/12 patients) } & \multicolumn{2}{|c|}{$R A$ (15 pregnancies/10 patients) } & \multicolumn{2}{|c|}{ Total (29 pregnancies $/ 22$ patients } \\
\hline & $\begin{array}{l}\text { Complicated } \\
(n=3)\end{array}$ & $\begin{array}{l}\text { Uncomplicated } \\
(n=11)\end{array}$ & $\begin{array}{l}\text { Complicated } \\
(n=4)\end{array}$ & $\begin{array}{l}\text { Uncomplicated } \\
(n=11)\end{array}$ & $\begin{array}{l}\text { Complicated } \\
(n=7)\end{array}$ & $\begin{array}{l}\text { Uncomplicated } \\
(n=22)\end{array}$ \\
\hline Anti-Ro $^{+}$ & 3 & 6 & 0 & 2 & 3 & 8 \\
\hline Anti-Ro- & 0 & 5 & 4 & 9 & 4 & 14 \\
\hline Anti-dsDNA ${ }^{+}$ & 3 & 5 & 0 & 0 & 3 & 5 \\
\hline Anti-dsDNA ${ }^{-}$ & 0 & 6 & 4 & 11 & 4 & 17 \\
\hline Anti-CL ${ }^{+}$ & 1 & 4 & 1 & 1 & 2 & 5 \\
\hline Anti-CL ${ }^{-}$ & 2 & 7 & 3 & 10 & 5 & 17 \\
\hline
\end{tabular}

mother with anti-Ro(SSA), anti-dsDNA, and anti$\mathrm{CL}$ antibodies. In the pregnancies of the patients with SLE the presence of anti-Ro(SSA) or antidsDNA antibodies was highly sensitive $(100 \%)$ but quite unspecific (specificity $45.5 \%$ and $54.5 \%$ respectively) in predicting pregnancy complicationthat is, premature deliveries and fetal losses). On the other hand, in these patients the presence of anti-CL antibodies was not a sensitive index of pregnancy complication (sensitivity $33.3 \%$ ) (Table 4). Of the four healthy controls with anti-CL antibodies (Table 2), a woman (aged 27) with IgM anti-CL had a history of one premature delivery and one spontaneous abortion. In addition, no autoantibody was found in two other women with multiple spontaneous abortions but also with full term deliveries.

\section{Discussion}

Pregnancy in autoimmune patients is a frequent clinical situation in the specialties of both obstetrics and rheumatology. The outcome of pregnancy can be affected by the presence of the autoimmune disease and vice versa. Therefore the interaction of these processes may be important for the health of both the mother and fetus. ${ }^{7}$
In comparison with healthy controls, women with SLE and MCTD were previously shown to have an increased incidence of total fetal loss-that is, the sum of fetal losses and therapeutic abortions-even before the clinical onset of their disease. ${ }^{3}$ Further, it has been suggested that the incidence of complicated pregnancies in these individuals increased after the development of clinical manifestations. ${ }^{36}$

In the present report we retrospectively evaluated the histories of a fairly large number of pregnancies of several unselected patients with ARD, including pregnancies before and after the disease onset. Our data indicate that compared with healthy controls women with ARD did not experience more frequently complicated pregnancies in general, or fetal loss in particular, either before or after the disease onset. As also previously described, ${ }^{26}$ however, prematurity (per pregnancy as well as per woman) was consistently more prevalent in our patients with SLE, both before and after their disease onset $(p<0.05)$, than in healthy controls. In addition, the incidence of stillbirths among pregnancies of patients with SLE increased significantly $(p<0 \cdot 05)$ after the disease onset, a fact also previously reported. ${ }^{36}$ It is noteworthy that, compared with data from some previous studies, complicated pregnancies in our patients with SLE were not as 
common. Previous reports on pregnancy outcome in SLE as well as in healthy women have indicated considerable variation in the prevalence of fetal loss, spontaneous abortions, stillbirths, and premature deliveries. ${ }^{3} 671415$ Reasons which may acount for these discrepancies include differences in patients and healthy control populations as well as in the severity and activity of the disease in the pregnant women examined.

In contrast with SLE, the course of pregnancy in patients with established diagnosis of RA or PSS is reported not to carry any particular fetal risk due to the presence of the disease. ${ }^{16} 17$ To our knowledge there are no data available on the pregnancy outcome in patients with RA or with $1^{\circ} \mathrm{SS}$ before the clinical appearance of their disease, while previously studied pregnancies before PSS did not show any remarkable incidence of complication. ${ }^{17}$ In this study it is shown that the incidence of spontaneous abortions (per pregnancy but not per women) in patients with PSS or $1^{\circ} \mathrm{SS}$ several years before their disease onset was significantly higher than in controls. We do not have a ready explanation for this observation, but it should be noted that these observed differences were of borderline statistical significance. Nevertheless, further studies are needed to clarify the issue.

The outcomes of the limited number of pregnancies before MCTD did not show any remarkable differences compared with controls. A previous study found that total fetal loss-that is, the sum of fetal losses and therapeutic abortions-was significantly higher in patients before MCTD. ${ }^{3}$ In that study, however, the incidence of fetal loss (termed fetal wastage) in patients before MCTD (16\% of pregnancies) did not differ from that of controls.

We could not show any association between the presence of autoantibodies under consideration, including anti-Ro(SSA), anti-dsDNA, and anti-CL antibodies, and any particular complication of pregnancy in the patients with ARD. In fact, a considerable percentage of pregnancies of anti-CL positive women (five out of seven) were completed as full term deliveries, suggesting limited, if any, predictive value of these autoantibodies for fetal risk. This finding, though in contrast with several reports, ${ }^{918}$ is in agreement with others. ${ }^{1920}$ In fact, the present data confirm our previous observations in unselected patients with ARD where, using an anti-CL assay shown to be highly specific and reproducible (by extensive preliminary experiments), we failed to show any association between the incidence of multiple abortions and the presence, the levels, or the isotypes of anti-CL antibodies. ${ }^{12}$ Apart from several methodological differences, which may account for the discrepancies between studies, ${ }^{12}$ multiple intrinsic or extrinsic factors, or both, including drug treatment and infections may be implicated in the miscarriages $\overrightarrow{=}$ encountered in patients with ARD. Therefore prospective studies are necessary to investigate further the prognostic significance of anti-CL anti- $\frac{\bar{c}}{\bar{D}}$ bodies for the outcome of pregnancy in unselected $\frac{\sqrt{\sigma}}{\vec{\phi}}$ patients with SLE and other autoimmune diseases.으 In addition, the influence of genetic and environ-ळ mental factors on the development of the anti-CL $\vec{\circ}$ associated manifestations remains to be determined.

In conclusion, our data indicate that subclinical $\vec{\omega}$ factors, which presumably may be present in patients with ARD before their disease onset, do noto appear to influence significantly the outcome of $\triangle$ pregnancies of these individuals.

We would like to thank Ms E E Papanikolaou for excellenก secretarial assistance.

\section{References}

1 Fraga A, Mintz G, Orazco J, et al. Sterility and fertility rates, fetal wastage and maternal morbidity in systemic lupus erythematosus. J Rheumatol 1974; 1: 293-8.

2 Mintz G, Niz J, Gutierrez G, et al. Prospective study of pregnancy in systemic lupus erythematosus. Results of a muifidisciplinary approach. J Rheumatol 1986; 13: 732-9.

3 Kaufman R L, Kitridou R C. Pregnancy in mixed connect tissue disease. Comparison with systemic lupus erythematosis. J Rheumatol 1982; 9: 549-55.

4 Moutsopoulos H M, Webber B L, Vlagopoulos T P, et al Differences in the clinical manifestations of sicca syndrome in the presence and absence of rheumatoid arthritis. $\mathrm{Am} \mathrm{J} \mathrm{Med \sigma o}$ 1979; 66: 733-6.

5 Masi A T, Rodnan G P, Medsger T A, et al. Preliminary criteria $\Rightarrow$ for the classification of systemic sclerosis (scleroderma). Arthri-음 tis Rheum 1980; 23: 581-90.

6 Gimovsky M L, Montoro M, Paul R H. Pregnancy outcome in? women with systemic lupus erythematosus. Obstet Gynecotó 1984; 63: 686-92.

7 Fine G L. Systemic lupus erythematosus in pregnancy. Ann Intern Med 1981; 94: 667-77.

8 Harris E N, Gharavi A E, Hughes G R V. Anti-phospholipid antibodies. Clin Rheum Dis 1985; 11: 591-609.

9 Lockshin M D, Druzin M L, Goei S, et al. Antibody to̊ cardiolipin as a predictor of fetal distress or death in pregnan patients with systemic lupus erythematosus. $N$ Engl J Med $1985^{\circ}$ 을
313: $152-6$.

10 Kelley W N, Harris E D Jr, Ruddy S, Sledge C B, eds. D Textbook of rheumatology. 2nd ed. Philadelphia: Saunders 1985.

11 Tzioufas A G, Manoussakis M N, Drosos A A, Silis G, Gharav A E, Moutsopoulos H M. Enzyme immunoassays for the detection of IgG and IgM anti-dsDNA antibodies: clinicab significance and specificity. Clin Exp Rheumatol 1987; 5 247-53.

12 Manoussakis M N, Gharavi A E, Drosos A A, Kitridou R Cৃ् Moutsopoulos H M. Anti-cardiolipin antibodies in unselected autoimmune rheumatic disease patients. Clin Immunol Im munopathol 1987; 44: 297-307.

13 Manoussakis $M$ N, Tzioufas A G, Silis M P, Pange P J E Goudevenos J, Moutsopoulos H M. High prevalence of anticardiolipin and other autoantibodies in a healthy elderl population. Clin Exp Immunol 1987; 69: 557-65. 
14 Devoe L D, Taylor R L. Systemic lupus erythematosus in pregnancy. Am J Obstet Gynecol 1979; 135: 473-9.

15 McCune A B, Weston W L, Lee L A. Maternal and fetal outcome in neonatal lupus erythematosus. Ann Intern Med 1987; 106: 518-23.

16 Neely $\mathrm{N} \mathrm{T}$, Persellin $\mathrm{R} \mathrm{H}$. Activity of rheumatoid arthritis during pregnancy. Tex Med 1977; 73: 59-63.

17 Ballon S P, Morley J J, Kushner I. Pregnancy and systemic sclerosis. Arthritis Rheum 1984; 27: 295-8.
18 Derue G, Englert H, Harris E N, et al. Fetal loss in systemic lupus erythematosus. Association with anti-cardiolipin antibodies. J Obstet Gynecol Neonatal Nurs 1985; 5: 207-9.

19 Stimmler M M, McGehee W G, Quismorio F P. A prospective study of anti-cardiolipin antibodies (ACA) in hospitalized SLE patients [Abstract]. Arthritis Rheum 1986; 29 (suppl): S44.

20 Petri M, Rheinschmidt M, Whiting-O'Keefe Q, et al. The frequency of lupus anticoagulant in systemic lupus erythematosus. Ann Intern Med 1987; 106: 524-31. 